\title{
Background light intensity and the pupillary response in a reaction time task
}

\section{JOHN L. BRADSHAW, Monash University, Victoria, Australia}

In an auditory reaction time task, changes in pupillary dilation were monitored during conditions of high and low background illumination. The latter were found to determine pupillary baseline levels, while the amplitude of the dilation peak at response stayed at a constant value. Unfulfilled expectancy that a response signal would occur was found to induce a smaller expectancy peak, despite the absence of an associated motor response. It was confirmed that temporal uncertainty relating to the length of the warning foreperiod could partly determine the $S_{s}^{\prime} R T$ performance.

In an earlier experiment it was shown (Bradshaw, in press) how an increase in stimulus uncertainty led to increased reaction time (RT), a rise in pupillary baseline levels, and an overall flattening of the associated response peaks. The degree of S's uncertainty was a function of the number of possible stimulus modalities, and the length or variability of a warning foreperiod. The pupillary reflex is of unusual interest, in that peripheral phenomena such as light intensity can partly determine it, as can certain central events involving attention, load, problem solving and information processing. Loewenfeld (1966) looked particularly at the photic determinants, while there have been a number of recent studies relating to the central aspects (e.g., Kahneman \& Beatty, 1966; Bradshaw, 1967).

The purpose of this experiment was to determine whether the magnitude of the pupillary phasic response is independent of the baseline levels within a wide but nonextreme range of illumination. The pupillary response was monitored during a RT task, and the baselines were preset by varying the background brightness.

\section{APPARATUS}

The photographic and recording apparatus has been described elsewhere (Bradshaw, 1968), as has the RT task and equipment (Bradshaw, in press). Briefly, an auditory warning signal was presented over earphones. This was followed by either a long or a short warning foreperiod of $5 \frac{1 / 2}{\mathrm{sec}}$ or $23 / 4 \mathrm{sec}$, respectively. The response signal was of slightly different pitch. The S's performance in closing a switch was measured to the nearest $1 / 100 \mathrm{sec}$ from the onset of the response signal. A marker was put on the recording film whenever any kind of signal or response occurred. Random alternations in long and short foreperiods were employed to avoid boredom and mechanical responding. For similar reasons instantaneous auditory feedback (a change in the frequency and continuation of the response signal) was provided as reinforcement on approximately half the sequences, whenever the S's RT exceeded an arbitarily set criterion. This criterion was set to the approximate mean of the S's overall RT hitherto, and was varied by the E accordingly.

The Ss viewed an evenly illuminated $24 \times 18$ in. back projection screen. Fixation was maintained upon a central point. The distance from screen to eye was 56 in., and the screen could be either bright or dark, the respective illuminations being $25 \mathrm{ft}-\mathrm{L}$ and $.56 \mathrm{ft}-\mathrm{L}$. Tangential illumination was provided from two $25-\mathrm{W}$ bulbs, each 12 in. from the left eye, one above and one below, on the temporal side of the $S$. The angle subtended on the eye by the two lamps was $50 \mathrm{deg}$. The angle subtended by either lamp and the line of fixation was $45 \mathrm{deg}$. The incident illumination from them measured at the eye, from a standard white surface occupying the latter's position, was $20 \mathrm{ft}-\mathrm{c}$ using an S.E.I. spot photometer. A voltage stabilizing circuit was employed throughout.

\section{PROCEDURE}

Seven Ss were employed, four female and three male. All were familiar with the apparatus. There was a counterbalanced order of presentation of the dark and light conditions, and the order of this counterbalancing itself alternated between Ss. Adaptation times of 7 min were allowed to elapse between conditions where illumination was changed. Prior practice up to performance asymptote was provided both before the experiment proper, and immediately before each new condition, after which recording commenced without a break.

The total cycle time or interval between successive warning signals was $15 \mathrm{sec}$. Each condition consisted of 12 such RT sequences. As each condition was represented twice in counterbalancing, this meant that for each condition, and for each S, there were 24 such sequences. Long and short warning foreperiods were randomly represented. Pupillary diameters were recorded at intervals of 2.7 per sec, giving 40 records per sequence. Diameters were measured to the nearest $.1 \mathrm{~mm}$. Because of the size of $\mathrm{N}$, subsequent averages were taken to $.05 \mathrm{~mm}$.

\section{RESULTS}

Figure 1 shows the averaged pupillary response curves for the two light conditions and foreperiod lengths. Taking as baseline the levels toward the tail ends of the two sets of curves, it can be seen that the dark condition imposes a baseline diameter increase of $33 \%$, or nearly twice the area. There is, however, little evidence of any change in peak amplitude or shape.

At the moment corresponding to when a response signal would have occurred after a short warning foreperiod, a small "expectancy" peak can be seen in the long foreperiod curves.

When the RT data were analyzed and averaged, it was found that the means were identical for the two conditions, light and dark. However, in all seven cases, the RT associated with a short warning foreperiod was greater than that associated with a long one. This proved significant at $p<.016$ by a two-tailed sign test, or $\mathrm{p}<.008$ if account is taken of the direction of this previously noted phenomenon (Bradshaw, in press).

As a consequence of this, it proved impractical to analyze the pupillary data for any emotive effect from the presence or absence of the reinforcement signal, because with all Ss there were many more successful longs and unsuccessful shorts than unsuccessful longs and successful shorts. However, inspection revealed no evidence of a trend, neither from the reinforcement signal subsequent to response, nor from intrinsically fast or slow responses themselves.

\section{DISCUSSION}

The occurrence of pupillary changes accompanying a motor response has been reported before (Nunnally et al, 1967), as it has in connection with problem solving. The present study is perhaps only the second one using a RT paradigm (cf. Bradshaw, in press). It is seen that an actual response is not necessary to induce a peak, as expectancy alone will often suffice, or merely the act of cognitive solution without overt verbalization (Bradshaw, 1967). In both cases the additional presence of a motor component increases the peak amplitude.

Within a single reflex system the interaction of sensory and autonomic processes was observed. While reduction of background illumination shifts the pupillary baselines by a factor nearly equal to one stop, no concurrent change was 


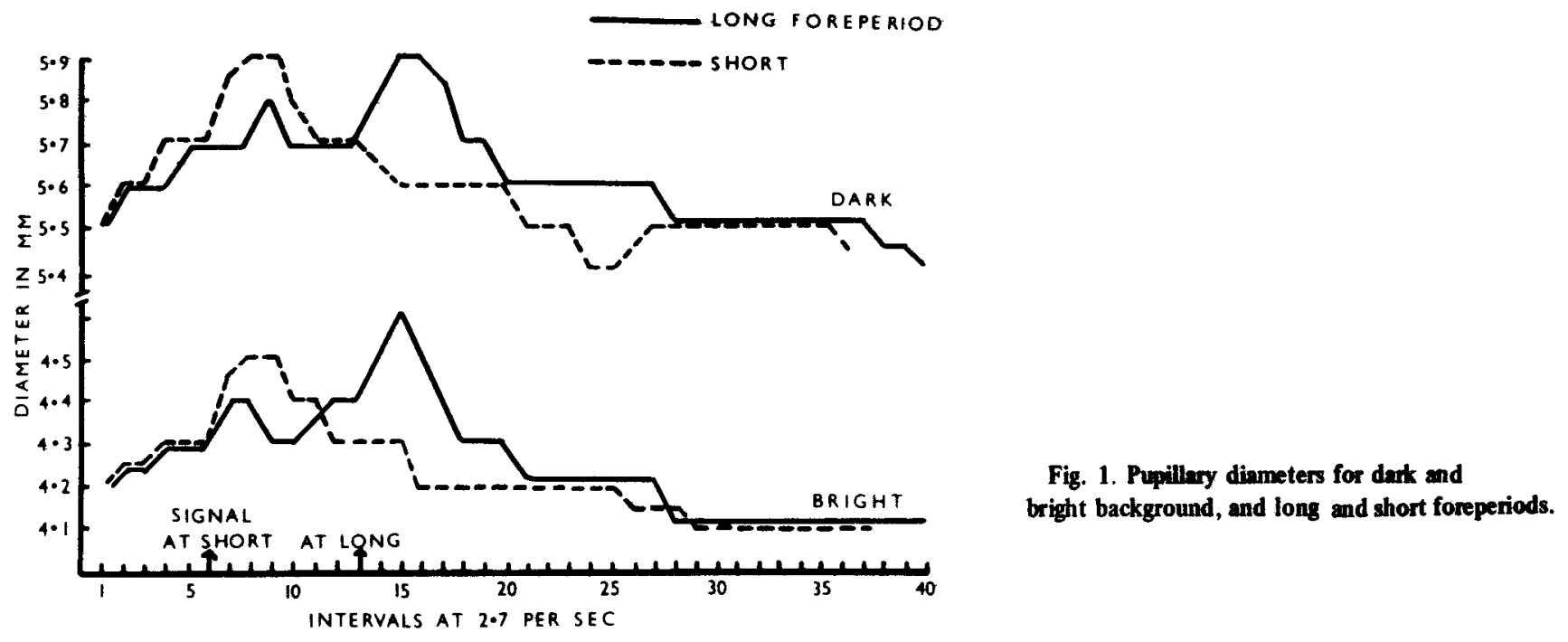

found in the amplitude of the peak effect. A problem which is ever present in the analysis of autonomic data is the extent to which baseline levels can determine the amplitude of a phasic effect, i.e., the Law of Initial Values (Lacey, 1956). In an unpublished study, Bradshaw compared the effects of alcohol and amphetamine upon pupillary levels in a RT task. Under alcohol, while the baselines were not affected, the response peak was dramatically reduced. Under amphetamine the baseline was considerably raised, without, however, any effect upon the response peak amplitude. Further research could usefully combine both background intensity and drug effects within the RT paradigm. With a complete absence of illumination to the eye, and further drug-induced baseline dilation, a rebound phenomenon might replace the response peak.

It was noted that longer warning foreperiods resulted in shorter RT. The effect of foreperiod length has been reviewed by Elliott (1964) and Fernandez-Guardiola et al (1968). If accuracy of time estimation was the only operative factor, one would expect a more accurate estimate, and swifter performance, with short foreperiods. However, it has been suggested (Bradshaw, in press) that at the end of a short foreperiod the $S$ does not know whether to expect a signal then or later. If, however, it does not then occur, he knows it will at the end of the long foreperiod. This will also account for the expectancy peak during the long foreperiod sequences, occurring at that moment corresponding to when a signal would otherwise have occurred.

While for reasons considered earlier, it proved impracticable to compare systematically the effect of the presence or absence of the reinforcement tone upon subsequent pupillary levels, there appeared to be little actual difference. This is perhaps an interesting finding in view of the Ss' reported gratification upon achieving such reinforcement, and the work of Hess (1965) concerning the effect on the pupillary response of emotively pleasing stimuli.

\section{REFERENCES}

BRADSHAW, J. L. Pupil size as a measure of arousal during information processing. Nature, 1967, 216, 515-516.

BRADSHAW, J. L. Load and pupillary changes in continuous processing tasks. British Joumal of Psychology, 1968, 59, 265-271.

BRADSHAW, J. L. Pupillary changes and reaction time with varied stimulus uncertainty. Psychonomic Science, in press.

ELLIOTT, R. Physiological activity and performance. Psychological Monographs: General \& Applied, 1964, 78, (10, Whole No. 587).

FERNANDEZ-GUARDIOLA, A.; AYALA, F., \& KORNHAUSER, S. EEG, heart rate and RT in humans: Variable vs fixed interval repetitive stimuli. Physiology \& Behavior, 1968, 3, 231-240.

HESS, E. Attitude and pupil size. Scientific American, 1965, 212, 46-54. KAHNEMAN, D., \& BEATTY, J. Pupil diameter and load on memory. Science, 1966, 154, 153-158.5.

LACEY, J. I. The evaluation of autonomic responses: Toward a general solution. Annals of the New York Academy of Sciences, 1956, 67, 123-163.

LOEWENFELD, 1. Pupillary movements associated with light and near vision: An experimental review of the literature. In $\mathbf{M}$. A. Whitcomb (Ed.), Recent developments in vision research. Washington: National Academy of Sciences, National Research Council Publication No. $1272,1966$.

NUNNALLY, J. C., KNOTT, P. D., DUCHNOWSKI, A., \& PARKER, R. Pupillary response as a general measure of activation. Perception \& Psychophysics, 1967, 2, 149-155. 